\title{
All-optical signal processing subsystems based on highly non-linear fibers and their limitations for networking applications
}

\author{
Miroslav Karasek ${ }^{1}$ \\ António Teixeira ${ }^{2}$, \\ Giorgio Tosi Beleffi ${ }^{3}$ \\ Ruben Luís ${ }^{2,3}$, \\ ${ }^{1}$ IREE, Prague - Czeck Republic \\ ${ }^{2}$ Instituto de Telecomunicações, Universidade de Aveiro, 3810-193 Aveiro Portugal' \\ ${ }^{3}$ ISCOM - Italian Communication Ministry Viale America n.201, Rome - Italy \\ ${ }^{4}$ Siemens SA, Amadora, Portugal \\ teixeira@ua.pt
}

\begin{abstract}
All optical signal processing is a fundamental concept on which the next generation networks will be based. Implementation of key functionalities as multiwavelength regeneration, frequency conversion and high data rate demultiplexing performed all optically becomes a primary target in current research scenario. In this work, demultiplexing of high data rate signals is demonstrated. The base for the functionality is the cross phase modulation occurring in a span of highly non linear fiber. This fiber is designed to have low birefringence, therefore quite adapted for processing polarization dependent processes, which are the base for the achieved functionality. Demultiplexing from $40 \mathrm{GHz}$ and $80 \mathrm{GHz}$ to $10 \mathrm{GHz}$ is demonstrated.
\end{abstract}

Keywords: OTDM, Demultiplexing, Cross Phase Modulation, Highly non linear fibers, supercontinuum, polarization multiplexing.

\section{Introduction}

Next generation networks (NGN) will be bring high bandwidth multimedia services closer to the end user, maintaining a reasonable per bit cost. Such structures will be fundamentally based on novel network topologies like for example 10 and 100 Gigabit Ethernet (GE) Passive Optical Networks (PON), technologies convergence like Radio over Fiber (RoF), Free Space Optics (FSO) and fiber based applications on the possibility to manage a huge amount of data adopting high speed rates and wavelength division multiplexing technologies.

In this scenario, the next key developments of these networks will be the provision of all optical network functionalities for the high bit rate network nodes. The avoidance of OEO conversions and the integration of functionalities at the nodes, determines the adoption of all optical techniques, which can be achieved with standard high non linear fibers or in photonic crystal fibers (PCF) [1,2]. As referred, some of these 
functionalities can be high bit rate wavelength conversion, for wavelength routing and collision avoiding, high bit rate time demultiplexing, for high speed WDM stream management, polarization multiplexing and signal reshaping.

All these functionalities can be achieved through all optical fast nonlinearities as cross-phase modulation (XPM), four wave mixing (FWM) and self phase modulation (SPM) in optical devices. Several approaches, in fact, have been proposed based on transferring the modulation to a carrier [3-5] or on broad spectrum generation with new carries [5], in semiconductor optical amplifiers [3] and optical fibers [3-6].

In this paper we present experimental results on high speed demultiplexing from 40 and $80 \mathrm{GHz}$ to $10 \mathrm{GHz}$ by means of XPM.

\section{Cross phase in highly nonlinear fibers}

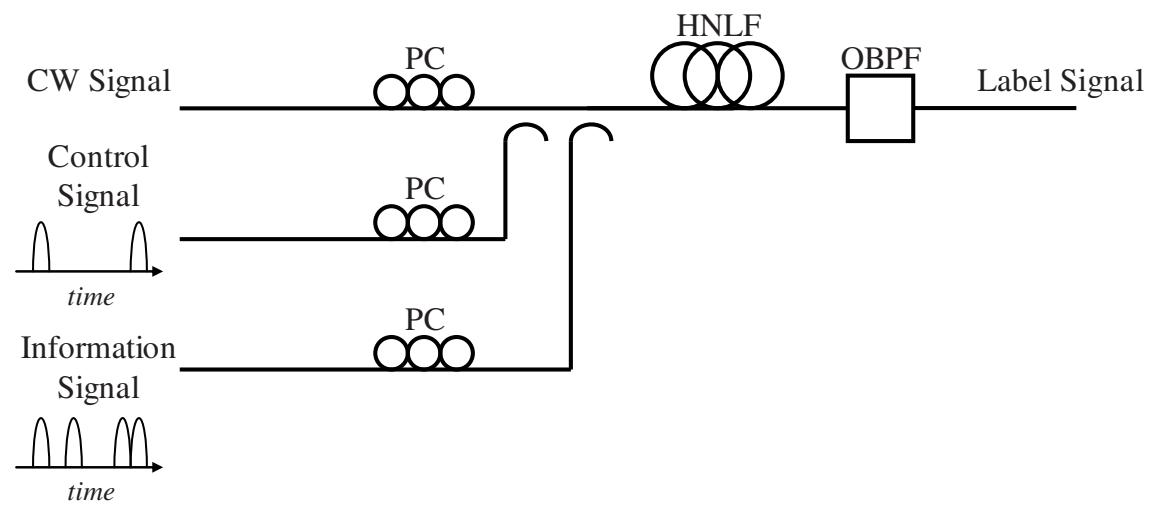

Fig. 1. All optical demultiplexing simulation set-up.

Consider the simulation set-up presented in Fig. 1. The $40 \mathrm{~Gb} / \mathrm{s}$ information signal is combined with an orthogonally polarized $10 \mathrm{~Gb} / \mathrm{s}$ impulse comb (control signal), positioned at a similar wavelength, and a continuous wave $(\mathrm{CW})$. It is assumed that the pulses of the control signal have the similar temporal width as the impulses of the information signal. The resulting signal is injected in a highly non-linear fiber (HNLF) segment. For simplicity we have omitted amplification stages that would be required to adjust the signal power. After the $\mathrm{HNLF}$, the $\mathrm{CW}$ signal is extracted by an off-centered optical band-pass filter (OBPF). Assuming orthogonally polarized information and control signals allows assuming that the XPM induced on the CW signal is dependent on a linear sum of the intensities of the interfering signals. As such, in a first order approach the XPM induced on the CW takes the form:

$$
\Delta \phi_{X P M}(t) \approx-2 \gamma\left[\eta_{s} \cdot p_{s}(t)+\eta_{c} \cdot p_{c}(t)\right] * h_{x p m}(t)
$$


where $p_{s}(t)$ and $p_{c}(t)$ are the instantaneous powers of the information and control signals and the symbol * denotes convolution. The terms $\gamma, \eta_{s}$ and $\eta_{p}$ are the HNLF non linear coefficient and the nonlinear coupling coefficients of the $\mathrm{CW}$ with respect to the information and control signals, respectively. $h_{x p m}(t)$ is the pulse response of the equivalent linear model for the cross-phase modulation. The XPM-induced instantaneous frequency shift may be obtained from (1) as $\Delta f(t)=\mathrm{d} \phi_{x p m} / \mathrm{d} t$. Fig.2 illustrates the XPM-induced instantaneous phase and frequency shifts at the HNLF output considering the control signal only, the information signal only and the combined signals.

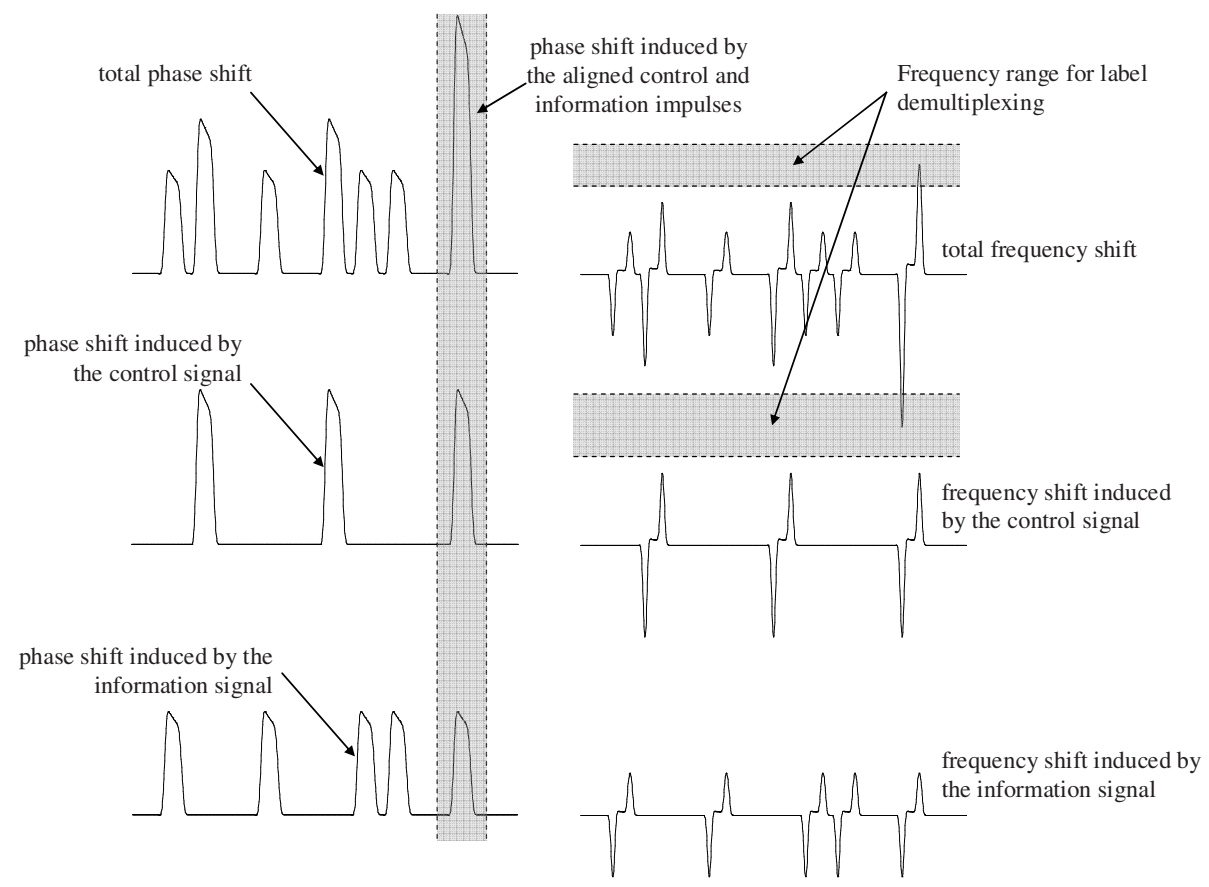

Fig. 2. Example of the XPM-induced instantaneous phase and frequency shifts of the CW signal, considering only the information signal (bottom), the control signal (middle) and both signals (top). The vertical axis of the XPM-induced phase shift has been reversed.

As shown in Fig.2, the alignment of an information impulse with a control impulse induces a particularly high phase shift. This generates a frequency shift significantly stronger that the phase shifts induced by the control or information signal alone. As such, one may define a range a frequencies within which the $\mathrm{CW}$ is positioned only when the control impulses superimpose the information pulses, as shown in Fig.2. Positioning the OBPF in this frequency range allows extracting the pulses from the original information signal. Note that nonzero dispersion and dispersion slope leads to asymmetric phase shifts. Therefore, in the presented example, the negative phase shifts are higher than the positive shifts. Fig.3 -a) and -b) present simulated traces and 
corresponding eye-diagrams of the signal at the OBPF output for two configurations of the latter. The simulated fiber parameters are presented in Table 1. We have assumed that the $\mathrm{CW}$ is co-polarized with the control signal. Furthermore, the OBPF output signal is detected using a simulated square-law receiver for $10 \mathrm{~Gb} / \mathrm{s}$, modeled by a 5 -th order Bessel filter with a $-3 \mathrm{~dB}$ cut-off frequency of $6.5 \mathrm{GHz}$.

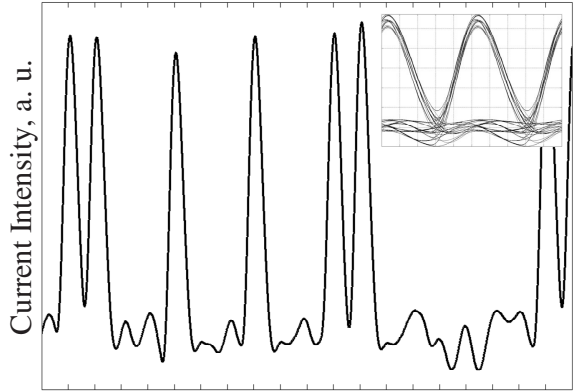

Time, $100 \mathrm{ps} / \mathrm{div}$

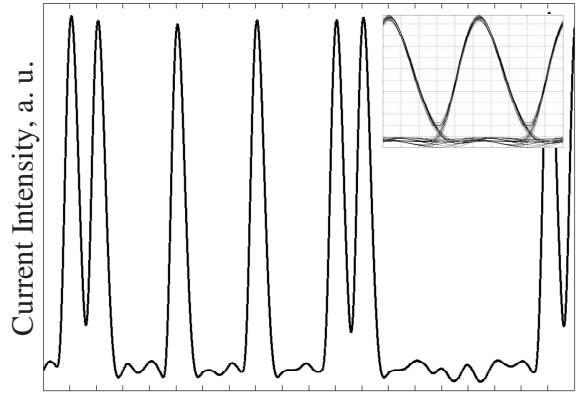

Time, $100 \mathrm{ps} / \mathrm{div}$

Fig. 3. Traces of the signal detected at the output of the OBPF. Left hand side: Filter off-center of $-460 \mathrm{GHz}$ and bandwidth of $250 \mathrm{GHz}$. Right hand side: Filter offcenter of $-460 \mathrm{GHz}$ and bandwidth of $100 \mathrm{GHz}$. Insets present the corresponding eye diagrams.

Fig.3 a) and -b) show the extracted signal affected by interference due to the control and signal impulses, particularly on the space level. Reducing the filter bandwidth from $250 \mathrm{GHz}$ to $100 \mathrm{GHz}$ significantly improves the signal quality at the cost of reducing its power by a factor of $\sim 10 \mathrm{~dB}$. The low power efficiency of this process may be attributed to the residual dispersion of the fiber and dispersion slope, which limit the XPM-induced frequency shift.

\section{Base Setup}

Several effects and dynamics in the area of Cross Phase Modulation (XPM) between a CW and a modulated signal responsible for the Supercontinuum generation were explored in this work. The CW, see Figure 1, is placed outside the SC spectrum generated from the self phase modulation of one u2t tunable pico-second source (PMLLD) at 10GHz. This PMLLD, can be multiplexed by an Optical Multiplexer (OTDM) to rates of 20, 40, 80 and $160 \mathrm{GHz}$. The HNLF characteristics are presented in table 1. 
Table 1. HNLF parameters.

\begin{tabular}{ll}
\hline Parameter & Value \\
\hline Zero dispersion wavelength & $1560 \mathrm{~nm}$ \\
Dispersion Slope & $0.019 \mathrm{ps} / \mathrm{nm}^{2} / \mathrm{km}$ \\
Nonlinear Coefficient & $10 \mathrm{~W}^{-1} \mathrm{~km}^{-1}$ \\
Length & $1500 \mathrm{~m}$ \\
Attenuation coefficient & $0.76 \mathrm{~dB} / \mathrm{km}$ \\
\hline
\end{tabular}

The possibility to obtain an all optical demultiplexer based on the above reported principles was also demonstrated. The principle behind this effect is the use of a 10 $\mathrm{GHz}$ pulsed stream at the same wavelength of the $40 \mathrm{GHz}$ stream, and by correct alignment induce differentiated XPM in the CW. This, after conversion to IM, will result in a process similar to demultiplexing. In order to avoid coherence beating effects, two distinct polarizations were used.

Figure 4 reports the experimental set up. The output of the PMLLD source, at 10 $\mathrm{GHz}$, has been amplified and subsequently split by means of an optical coupler. The same signal is, by means of this double arm structure, one part guided to an OTDM in order to obtain a $40 \mathrm{GHz}$ signal and the other send to a delay and amplitude adjustment arm. The signals in the two arms are polarization rotated to maximize the alignment with the Polarization beam combiner (PBC). Due to the fact that both signals originate from the same source they have the same working wavelength, $1556.8 \mathrm{~nm}$. The orthogonally polarized signals arrive at the HNLF, after amplification, along with the auxiliary CW carrier (Figure 2A).

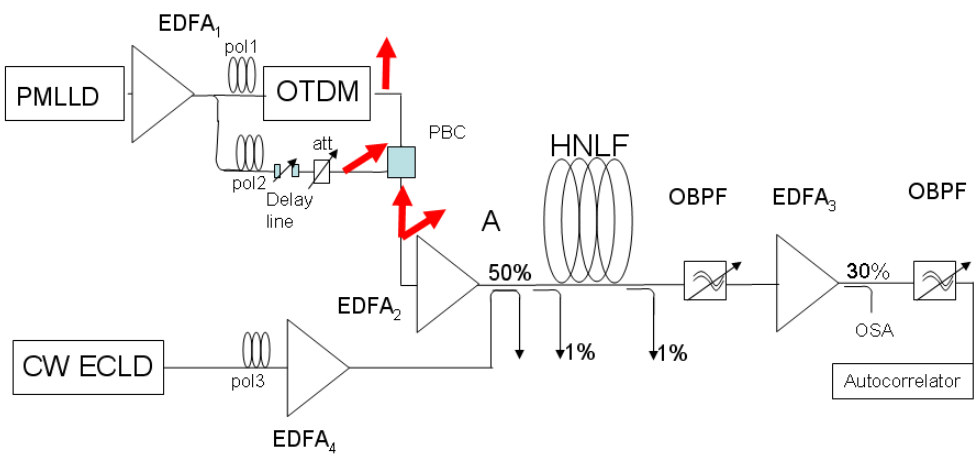

Fig. 4. All optical demultiplexing experimental Set-Up

In Figure 5, the HNLF input spectrum, $\mathrm{CW}$ and $40 \mathrm{GHz}+10 \mathrm{GHz}$ signals (A), and the Autocorrelator trace of the signal at $40 \mathrm{GHz}(\mathrm{B})$ are been reported. 

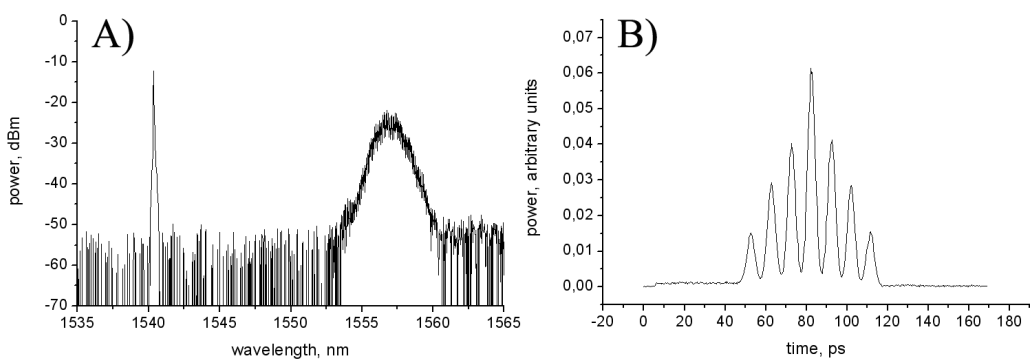

Fig. 5. A) HNLF input spectrum. B) Autocorrelator trace

In Figure 6 the output of the HNLF is reported. In particular, it can be noticed that when the $10 \mathrm{GHz}$ signal is shut off, the $40 \mathrm{GHz}$ modulation on the auxiliary $\mathrm{CW}$ carrier becomes more evident (Figure 3B).
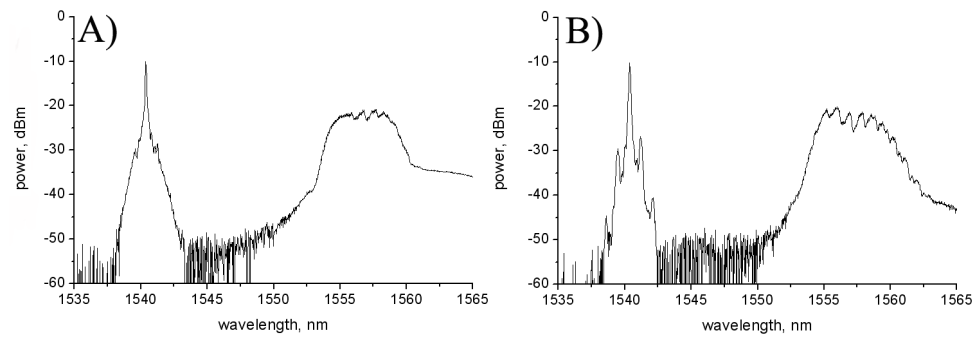

Fig. 6. Optical spectrum at HNLF output: A) with $40 \mathrm{GHz}$ signal and $10 \mathrm{GHz}$ demultiplexing stream. B) the $40 \mathrm{GHz}$ signal alone.

Adjusting the power of the $10 \mathrm{GHz}$ stream and moving the delay line is possible to observe the demultiplexing efficiency effect. From A) to D) (Figure 7) the delay line is moved always in the same direction. Figure $4 \mathrm{C}$ is reflecting what we can assume to be the deumultiplexing situation, since there is only one peak, which corresponds, due to the autocorrelator time window, to the perfect overlap between the $10 \mathrm{GHz}$ demultiplexing stream and one of the 4 multiplexed pulses of the $40 \mathrm{GHz}$. All the other situations show the $40 \mathrm{GHz}$ residual stream (Figure 7A, B and D).

The concept behind the demultiplexing willing to be achieved with this setup, is the linear dependency of the nonlinear phase on the power of the XPM inducing signal. This higher power of the latter, the higher is the nonlinear phase shift suffered by the CW. Also, since coherence is a problem which could endanger the process, two polarizations were used. By formatting the filter shape and central position, one can control the XPM part which is extracted. In the experiments a simple off the shelf filter was used, therefore limiting the overall result. However, the same situation as described for $40 \mathrm{GHz}$, was achieved with a $80 \mathrm{GHz}$ pulse stream. Another effect which will certainly be of interest are the time delays and amplitude difference between the $10 \mathrm{GHz}$ demultiplexing stream and the stream to be demultiplexed. The advantage of having the two in the same wavelength is that the walkoff is minimized, increasing therefore the efficiency of the process. 

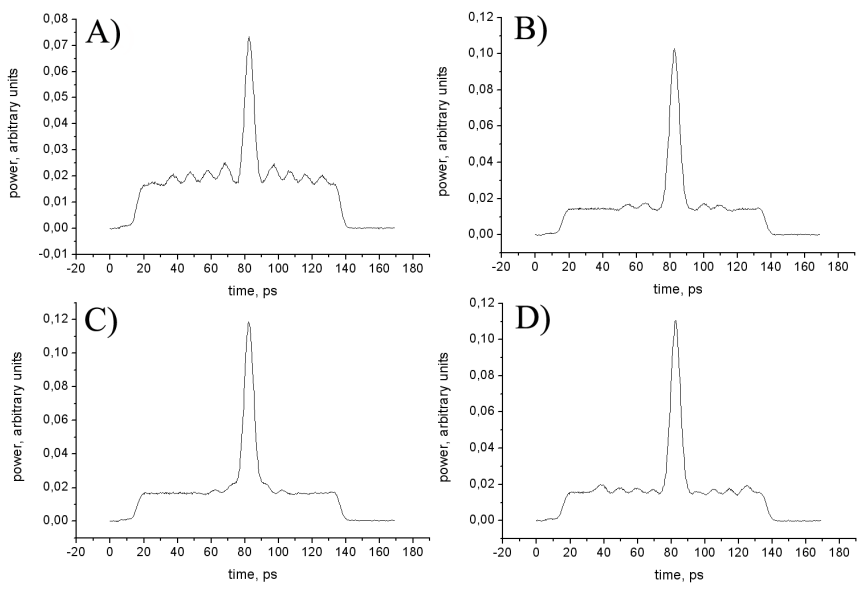

Fig. 7. A) B) C) D) Different positions of the delay line. In C) we have a good overlapping of the pulses and so a good demultiplexing from $40 \mathrm{GHz}$ to $10 \mathrm{GHz}$.

In Figure 8 are presented the results for several relative delays between the $80 \mathrm{GHz}$ stream to be demultiplexed and the $10 \mathrm{GHz}$ signal. A moderately good result was achieved, given the fact that the filter shape was not changed, therefore probably not optimum for demultiplexing at this frequency rate.
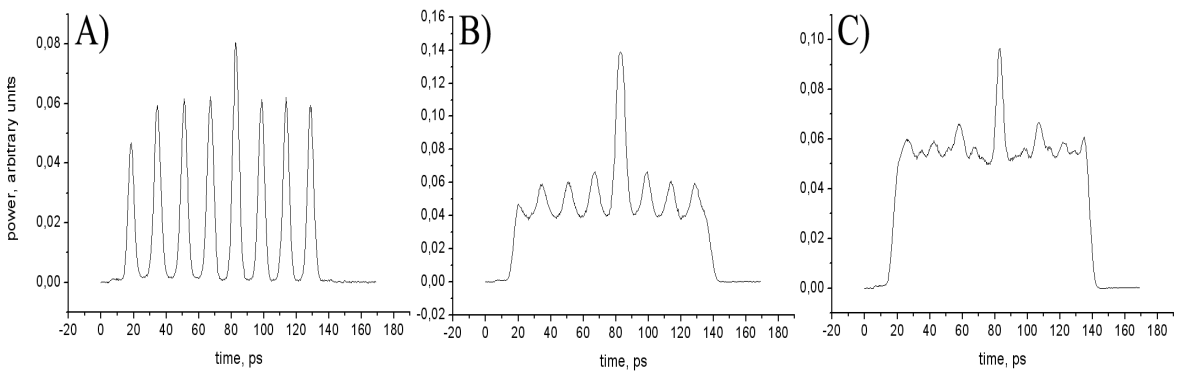

Fig. 8. A) $80 \mathrm{GHz}$ input. B) and C) two different delays

In this process it is important to have a convenient choice of the powers of the two streams in order to maximize the effect.

\section{Conclusions}

Cross phase modulation occurring in high non linear optical fibers based on single wavelength orthogonal state of polarization has been used to achieve all optical demultiplexing. The results demonstrates that $40 \mathrm{GHz}$ and $80 \mathrm{GHz}$ to $10 \mathrm{GHz}$ demultiplexing can be easly achieved in a simple and scalable way. This technique furthermore suggests that good results could be obtained at even higher rated if tailored filters and suitable dispersion maps are used. 
Acknowledgments. This work was supported by the project Cost 291. The authors would also like to thank the IREE for receiving us and the projects NoE e-Photon $\mathrm{ONe}+$ and CONPAC (POSC/EEA-CPS/61714/2004) for the complementary support.

\section{References}

1 Nolting, H.-P.; Sartorius, B. "Advanced devices and their features for future optical networks", LEOS 2000. 13th Annual Meeting. IEEE Volume 2, 13-16 Nov. 2000 Page(s):627 - 628 vol.2

2 Russell, P. St.J.; "Photonic-Crystal Fibers" Lightwave Technology, Journal of Volume 24, Issue 12, Dec. 2006 Page(s):4729 - 4749

3 Y. Ueno et al "Penalty-free error-free all-optical data pulse regeneration at $84 \mathrm{~Gb} / \mathrm{s}$ by using a symmetric-Mach-Zehnder-type semiconductor regenerator," IEEE Photon. Technol. Lett., vol. 13, no. 5, pp. 469-471, May 2001.

$4 \mathrm{~S}$. Murata et alt "THz optical-frequency conversion of $1 \mathrm{~Gb} / \mathrm{s}$-signals using highly nondegenerate four-wave mixing in an InGaAsP semiconductor laser," IEEE Photon. Tech. Lett., vol. 3, pp. 1021-1023, Nov. 1991.

5 Bengt-Erik Olsson, Daniel J. Blumenthal et alt, " A Simple and Robust 40-Gb/s Wavelength Converter Using Fiber Cross-Phase Modulation and Optical Filtering", IEEE Phot. Technol. Lett., Vol.12, No.7, JULY 2000

6 P. V. Mamyshev, "All-optical data regeneration based on self-phase modulation effect," in Proc. Eur. Conf. Optical Communication (ECOC'98), Madrid, Spain, Sep. 20-24, 1998, pp. 475-476.

7 S. Taccheo and P. Vavassori, "Dispersion-flattened fiber for efficient supercontinuum generation", paper ThU5, OFC 2002, Anaheim, CA, USA (2002) 